\title{
Space Propagation Characteristics of Audible Noise Due to HVDC Transmission Lines
}

\author{
Shao Hua ${ }^{1}$, Hou Shanshan ${ }^{2}$
}

State grid of Anhui electric power company maintenance company Hefei, China 230000

Keywords: audible noise; HVDC transmission line; atmospheric absorption; spectrum characteristics

Abstract: Audible noise generated by the corona of HVDC transmission line has become one of the major considerations in the design of high voltage transmission lines. To research audible noise space propagation characteristics duo to HVDC transmission lines is very important for the analysis of the spatial frequency spectrum characteristics of the audible noise, and it can provide the basis for the transmission line audible noise environment. Absorption attenuation of expression, and expression as well as the atmosphere of the semi-free-space point source sound pressure wave superposition principle to calculate the space of a HVDC transmission line audible noise spectrum distribution. Then discuss the spatial propagation characteristics of high voltage direct current transmission line audible noise under different weather conditions. The analysis results show that: As the the atmospheric absorption attenuation, HVDC transmission line audible noise spectrum in the case of low-frequency, but a greater impact in the high-frequency case.And under different weather conditions, the high-frequency part of the spectrum changes Larger.

\section{Foreword}

With the rapid development of power grid construction, building direct current transmission line voltage level higher and higher, and with the improvement of people's living standards, awareness of environmental protection has been strengthened ${ }^{[1]}$. so that the problem of audible noise generated by the DC transmission line corona becomes one of the factors DC transmission line construction and planning must be considered. Therefore,study on the propagation characteristics of space audible noise generated by the DC transmission line corona, audible noise for accurate prediction of the DC transmission lines as well as the subsequent construction of transmission lines has an important role ${ }^{[2]}$.

Audible noise of generated DC transmission line is mainly due to transmission line effects resulted in the molecular layer vibration corona space propagation. DC transmission line corona audible noise generated mainly from the positive polarity streamer discharge, and therefore the DC line only need to consider audible noise generated by the positive lead. Then, based on the wave's principle of superposition transmission line will generate audible noise is regarded as a large number of uniformly distributed point source superimposed ${ }^{[3]}$.

\section{Point source computing in space attenuation characteristics of the communication process}

Point source in half free space propagation process if you do not include the attenuation caused by atmospheric absorption, Then the sound of the measured points from point source of pressure is:

$$
P=P_{0}\left[\left(r_{0} \times e^{-j k\left(r-r_{0}\right)} / r\right)+\right.
$$




$$
\left.R_{h}\left(r_{0} \times e^{-j k\left(r_{1}-r_{0}\right)} / r_{1}\right)\right]
$$

The $\mathrm{R}$ is reflection coefficient

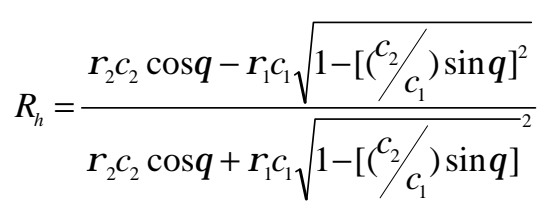

Where: $r$ is the direct sound from; $r_{1}$ is the reflected sound from; $r_{0}$ Known point to the the sound source distance from; $\rho_{1}$ is air density; $\rho_{2}$ is ground density; $c_{1}$ is Air Velocity; $c_{2}$ is ground speed of sound; $\theta$ is angle of Incidence; $P_{0}$ is sound pressure amplitude of the known points. Half the size of the sound pressure point source in free space, we can see that the point source in the SPL measured point is:

$$
L_{p}=20 \log 10\left(|p| / 20 \times 10^{-6}\right)
$$

$|p|$ is Sound pressure amplitude of measured point; $L_{p}$ is SPL of measured points.

\section{Atmospheric attenuation effect on the DC transmission line}

In this paper, by assuming different environment variable analysis of atmospheric attenuation effect on the spatial characteristics of the DC transmission line of audible noise. Assuming a large number of point sources such as power transmission line spacing for the same phase composition. DC transmission line length of 300 meters and a height of 25 meters on the ground, the selected measurement point is 1.5 meters above the ground, measuring line perpendicular to the line of projection. DC transmission line space atmospheric attenuation amount shown in Figure 1

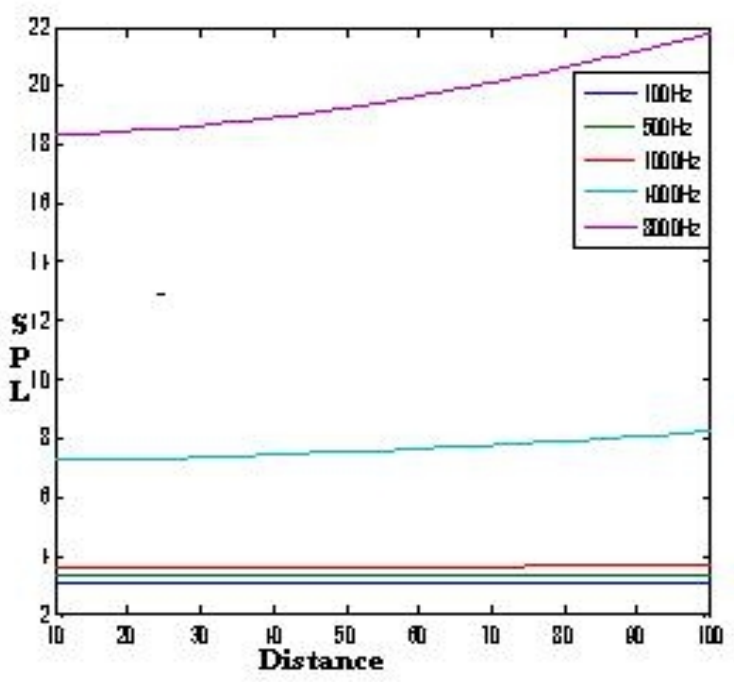

Fig.1 DC transmission line to absorb different frequencies audible noise attenuation of lateral distribution of atmospheric

DC transmission line audible noise in the communication process space ${ }^{[4]}$, the attenuation due to atmospheric absorption, resulting in attenuation of the sound pressure level. At low frequencies ( $\leqq 400 \mathrm{~Hz}$ ) case, the DC transmission line atmospheric attenuation is very weak, but with 
increasing frequency, especially when the frequency is increased under high frequency ( $\geqq$ $1000 \mathrm{~Hz}$ ) case, the DC transmission line in space dissemination of attenuation by the atmospheric absorption has become particularly pronounced attenuation of the DC transmission line at this time is

particularly large. Analysis and comparison of DC transmission line in the process of space spread divergent, and consider the transmission line attenuation and atmospheric absorption interaction of geometric divergence difference.

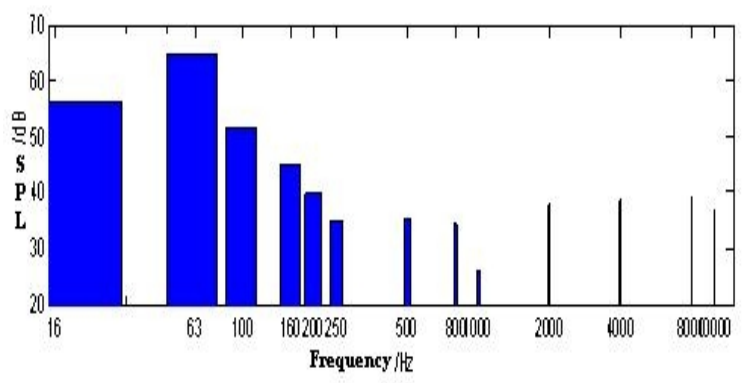

(a) direct current transmission lines without considering the atmospheric absorption spectrum

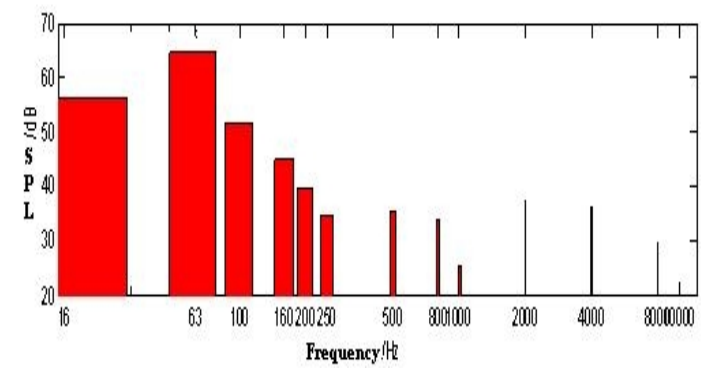

(b) direct current transmission line to consider the atmospheric absorption spectrum

Fig.2 transmission line audible noise spectrum

Figure 2 shows the comparison: HVDC transmission line audible noise in the low-frequency part of the role of atmospheric attenuation little change, and with increasing frequency, especially in the high-frequency part of the atmospheric attenuation has a relatively large amount, so a-weighted sound pressure levels cause the entire HVDC transmission lines under consideration in the case of atmospheric attenuation does not consider the situation to be less than atmospheric absorption attenuation.

\section{DC transmission line audible noise attenuation changes under different weather conditions analysis}

Assuming standard atmospheric pressure, the DC line length of 300 meters, a height of 25 meters on the ground, the selected measurement point is 1.5 meters above ground, the measuring point for the projection of the perpendicular distance from the line 20 meters. When the pressure remains constant (atm), the DC transmission line near ambient temperature of $20{ }^{\circ} \mathrm{C}$, relative humidity of the atmosphere changed, audible noise spectral distribution shown in Figure 3. 


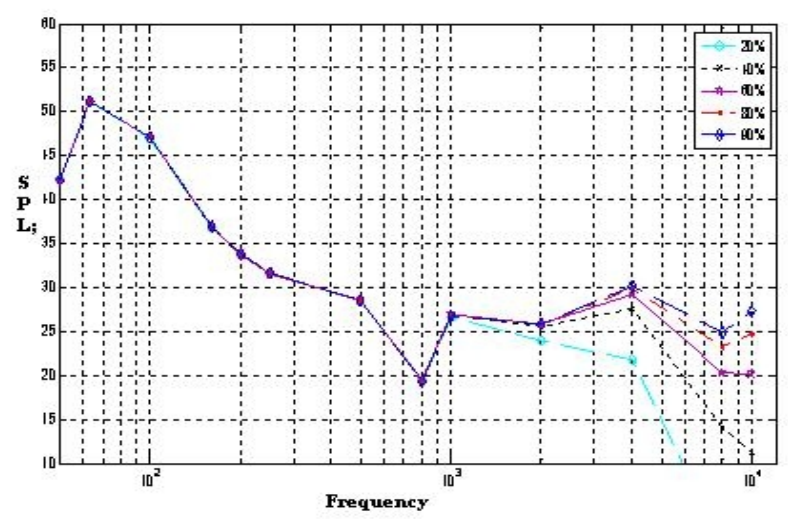

Fig.3 temperature is $20^{\circ} \mathrm{C}$, the DC transmission line audible noise spectrum with the relative humidity changes

Figure 3 shows, the DC transmission line audible noise emitted during propagation in space, at a distance of 20 meters in the vertical outer conductor when pressure remains constant (standard atmospheric pressure) and a temperature of $20{ }^{\circ} \mathrm{C}$, the audible noise increases as the relative humidity, the low frequency portion of the spectrum changes substantially unchanged, with the high frequency portion of the sound pressure level relative humidity increases.

Conditions as above, maintaining the standard atmospheric pressure, relative humidity of $60 \%$, temperature from $-20{ }^{\circ} \mathrm{C}$ to $10{ }^{\circ} \mathrm{C}$ increments to $30{ }^{\circ} \mathrm{C}$, the audible noise spectrum changes with temperature as shown in Figure 4.

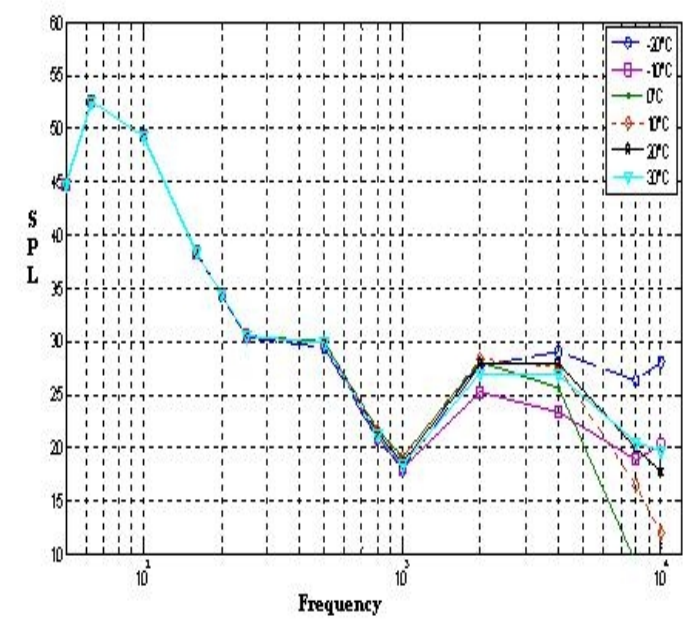

Fig.4 relative humidity is $60 \%$ of the DC transmission line audible noise spectrum changes with temperature

From the above chart we can see that the standard atmospheric pressure, relative humidity of $60 \%$, the DC transmission line audible noise in the low frequency not change with temperature, but the high-frequency part of the time at a lower temperature the smaller the amount of attenuation of atmospheric absorption.

\section{Summary}

In this paper, the sound of the superposition principle deduced DC transmission line audible noise space propagation model, and then create a DC transmission line audible noise in the space containing the spread of atmospheric absorption attenuation model. In the calculation assuming DC transmission line is a single wire structure. Discusses the DC transmission line audible noise attenuation contains effects of atmospheric absorption spectrum of its time, and calculated in the same atmospheric pressure, temperature and relative humidity changes, the DC transmission line audible noise in the measured point spectrum . 


\section{References}

[1] Liu Zhenya. UHV project electromagnetic environment[M]. China Electric Power Press. 2009

[2] Si Wen. Electromagnetic Environmental Research HVDC transmission lines and AC-DC Parallel. Shandong University Thesis.

[3] P.Sarma Maruvada. Corona performance of high-voltage transmission lines [M]. Research tudies spress LTD.

[4] T. F. W. Embleton, J. E. Piercy, G. A. Daigle. Effective flow resistivity of ground surfaces determined by acoustical measurements [J]. Acoustical Society of America, 1983, 74(4): 1239-1244.

[5] ISO 9613-1:1993, Acoustics-Attenuation of sound during progaration outdoors-Part 1:Calcultion of the absorption of sound by the atmosphere. 